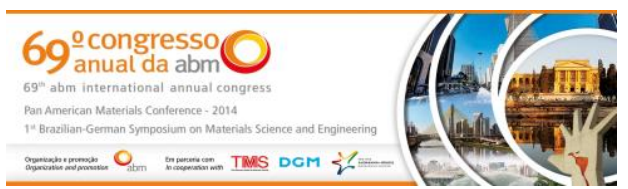

Tema: Metalurgia física e comportamento de materiais em temperaturas elevadas

\title{
ACOMPANHAMENTO DA FORMAÇÃO DE FASE SIGMA NO AÇO INOXIDÁVEL DUPLEX SAF2205 (UNS31803) POR RUÍDO MAGNÉTICO DE BARKHAUSEN (MBN)*
}

\author{
Edgar Apaza Huallpa ${ }^{1}$ \\ Eduardo Franco de Monlevade ${ }^{2}$ \\ Manuel Alberteris ${ }^{3}$ \\ Linilson R. Padovese ${ }^{4}$ \\ Hélio Goldenstein ${ }^{5}$
}

\section{Resumo}

Os aços inoxidáveis duplex (AID), apresentam em geral uma microestrutura composta de austenita e ferrita em proporções aproximadamente iguais. Para ter o controle dos processos de fabricação e desempenho é importante conhecer sua evolução microestrutural, devido à precipitação de fases intermetálicas como sigma $(\sigma)$ e chi $(X)$, que causam grave deterioração das propriedades mecânicas do material. Neste trabalho foi estudada a evolução da fase sigma $(\sigma)$ durante tratamentos térmicos em temperaturas intermetálicas $\left(800^{\circ} \mathrm{C}-900^{\circ} \mathrm{C}\right)$ de amostras do aço inoxidável duplex tipo SAF2205 (DIN 1.4462/UNS 31803). Foi observada uma gradual redução da intensidade do sinal do ruído de Barkhausen com o aumento do tempo de tratamento térmico, indicando uma redução na fração de fase ferromagnética. Para tempos de tratamento de 24 horas, o sinal de Ruído de Barkhausen está quase totalmente inserido dentro do ruído de fundo da medida, indicando haver uma fração volumétrica bastante reduzida de ferrita. Por meio da produção de amostras padrão para calibração, essa pode ser uma via de avaliação não destrutiva de componentes submetidos em campo a temperaturas nas quais se formam fases intermetálicas.

Palavras-chave: Ruído magnético Barkhausen; Aços inoxidáveis dúplex; Fase sigma; Ensaios não destrutivos.

\section{USE OF MAGNETIC BARKHAUSEN NOISE (MBN) TO FOLLOW UP THE FORMATION OF SIGMA PHASE IN SAF2205 (UNS31803) DUPLEX STAINLESS STEEL \\ Abstract}

Duplex stainless steels have a structure normally composed of auteite and ferrite in approximately equal proportions. In order to attain control of its fabrication processes and performance, it is important to understand its microstructural evolution, due to the formation of intermetallic phases such as sigma $(\sigma)$ and chi $(X)$, that may cause a severe deterioration of the mechanical properties of duplex stainless steels. In the present study, the evolution of sigma phase during heat treatments at temperatures in which intermetallic phases cam be formed $\left(800^{\circ} \mathrm{C}-900^{\circ} \mathrm{C}\right)$ was studied in a SAF2205 (DIN 1.4462/UNS31803) steel. A significant reduction of the intensity of Magnetic Barkhausen Noise (MBN) was observed with the increase of heat treatment time, indicating a decrease in the quantity of ferromagnetic phases. For 24-hour-long treatments, the Barkhausen Noise signal is almost completely enclosed by the background noise, indicating the existence of a very small volume fraction of ferrite. If proper calibration samples are to be produced, this technique may be a viable method for non-destructive evaluation of field components working under thermal conditions that may cause the formation of intermetallic phases.

Keywords: Magnetic Barkhausen noise; Duplex stainless steels; Sigma phase; Nondestructive testing

\footnotetext{
Doutorando, Depto. Engenharia Metalúrgica e de Materiais, Escola Politécnica, USP, São Paulo, SP, Brasil. Prof. Doutor, Depto. Engenharia Metalúrgica e de Materiais, Escola Politécnica, USP, São Paulo, SP, Brasil. Pós-doutorando, Depto. de Engenharia Mecânica, Escola Politécnica, USP, São Paulo, SP, Brasil. Professor Associado, Depto. de Engenharia Mecânica, Escola Politécnica, USP, São Paulo, SP, Brasil. Professor Titular, Depto. Engenharia Metalúrgica e de Materiais, Escola Politécnica, USP, São Paulo, SP, Brasil.
}

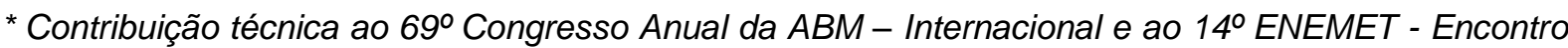
Nacional de Estudantes de Engenharia Metalúrgica, de Materiais e de Minas, 21 a 25 de julho de 2014, São Paulo, SP, Brasil.
} 


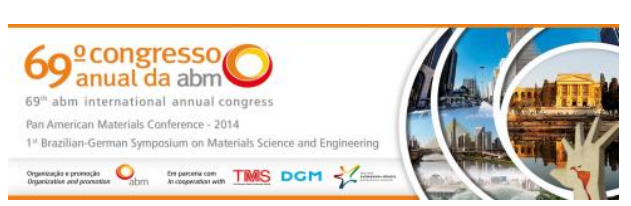

\section{INTRODUÇÃO}

Operações de soldagem e conformação a quente podem produzir transformações microestructurais indesejáveis nos AID como as fases sigma $(\sigma)$, chi $(X)$ ou nitretos de cromo ( $\mathrm{Cr} 2 \mathrm{~N})$. Devido a que a fase sigma é paramagnética pode ser estudada por medidas magnéticas.

Ruído magnético de Barkhausen (MBN) baseia-se na deteç̧ão por indução de um sinal gerado em matérias ferromagnéticas submetido a um campo magnético externo, onde as paredes de domínio magnético são forcadas a recuar e avançar, quando são submetidas por um campo magnético externo oscilante.

A mudança no campo magnético sofre resistência devido a barreiras dissipativas no movimento das paredes de Bloch. Assim, quando um grupo de paredes de domínio finalmente muda, é de forma abrupta e irreversível. A energia interna do sistema atinge um nível alto, causando a emissão de picos repentinos ou pulsos de campo magnético, gerando pulsos de voltagem numa bobina de sensor colocado na superfície da amostra.

Estes pulsos são chamados MBN em honra de Heinrich Barkhausen, que descobriu este fenômeno em 1919.

Precipitados, contornos de grão, discordâncias e campos de tensão atuam como barreiras efetivas para o movimento da parede de domínio de modo que MBN é sensível à microestrutura, deformação elástica e plástica em materiais ferromagnéticos $[1,2]$. Também é fortemente influenciada pelos defeitos da estrutura, tais como poros, inclusões de segunda fase, discordância, ancoragem de imperfeições pontuais e tensões no material [3].

\section{MATERIAIS E MÉTODOS}

Para este estudo foram utilizadas amostras do aço inoxidável duplex do tipo DIN 1.4462 (UNS 31803).

\subsection{Duplex DIN 1.4462 (UNS 31803)}

Os aços inoxidáveis Duplex são uma família que combina uma boa resistência à corrosão com alta resistência e facilidade de fabricação, apresentam uma microestrutura mista com proporções similares de austenita e ferrita, existem há aproximadamente 80 anos.

Composição química (Tabela 1).

\footnotetext{
* Contribuição técnica ao $69^{\circ}$ Congresso Anual da ABM - Internacional e ao 14ํㅡㄹ ENEMET - Encontro Nacional de Estudantes de Engenharia Metalúrgica, de Materiais e de Minas, 21 a 25 de julho de 2014, São Paulo, SP, Brasil.
} 
Tabela 1: Composição química (\% em peso)

\begin{tabular}{|c|c|}
\hline Elemento & $\%$ em peso \\
\hline $\mathrm{C}$ & 0,0160 \\
\hline $\mathrm{Si}$ & 0,6600 \\
\hline $\mathrm{Mn}$ & 0,6200 \\
\hline $\mathrm{P}$ & 0,0090 \\
\hline $\mathrm{S}$ & 0,0016 \\
\hline $\mathrm{Co}$ & 0,0300 \\
\hline $\mathrm{Cr}$ & 22,6000 \\
\hline $\mathrm{Mo}$ & 3,0600 \\
\hline $\mathrm{Ni}$ & 4,7300 \\
\hline $\mathrm{V}$ & 0,0200 \\
\hline $\mathrm{Cu}$ & 0,0700 \\
\hline $\mathrm{Al}$ & 0,0130 \\
\hline $\mathrm{B}$ & 0,0031 \\
\hline $\mathrm{N}$ & 0,2000 \\
\hline
\end{tabular}

\subsection{Temperaturas dos tratamentos térmicos}

Foi calculado o mapa de fases utilizando o Thermo-Calc®, para escolher as temperaturas para os tratamentos térmicos. Nos cálculos foram levados em conta os elementos de liga: $\mathrm{Fe}, \mathrm{Cr}, \mathrm{Ni}, \mathrm{Mo}, \mathrm{Cu}, \mathrm{N}, \mathrm{C}, \mathrm{Si}$ e $\mathrm{Mn}$; e as fases: Liquido, ferrita austenita, $\mathrm{Cr}_{2} \mathrm{~N}$, Sigma e $\mathrm{M}_{23} \mathrm{C}_{6}$.

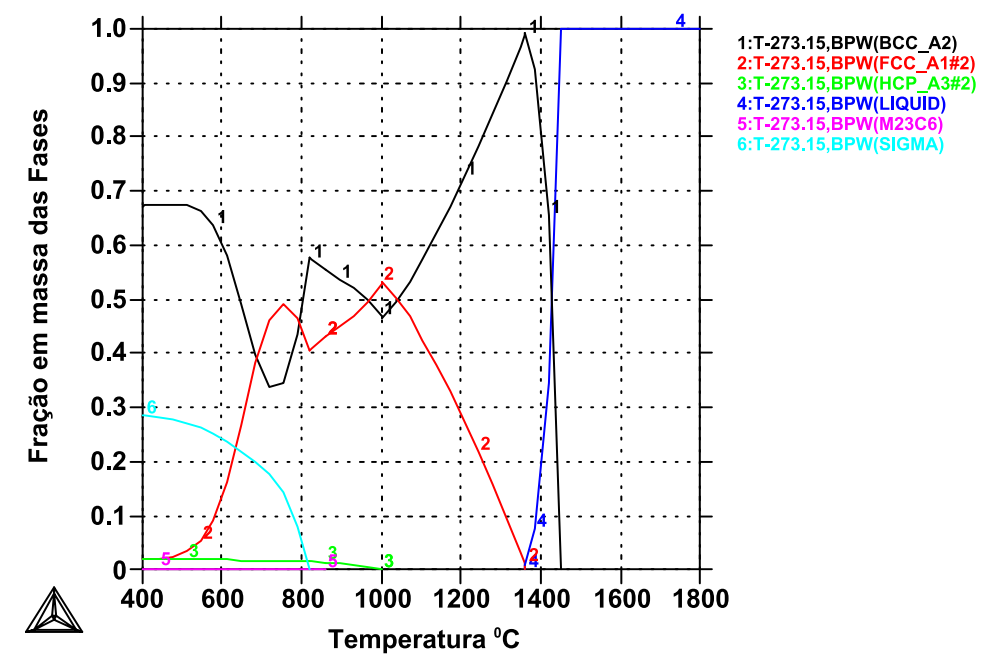

Figura 1 - Mapa de fases calculado com o programa Thermo-Calc® aliado ao banco de dados TCFE. Nas ordenadas a escala é linear (fração mássica das fases).

\footnotetext{
* Contribuição técnica ao 69ำ Congresso Anual da ABM - Internacional e ao 14ํㅡㄹ ENEMET - Encontro Nacional de Estudantes de Engenharia Metalúrgica, de Materiais e de Minas, 21 a 25 de julho de 2014, São Paulo, SP, Brasil.
} 

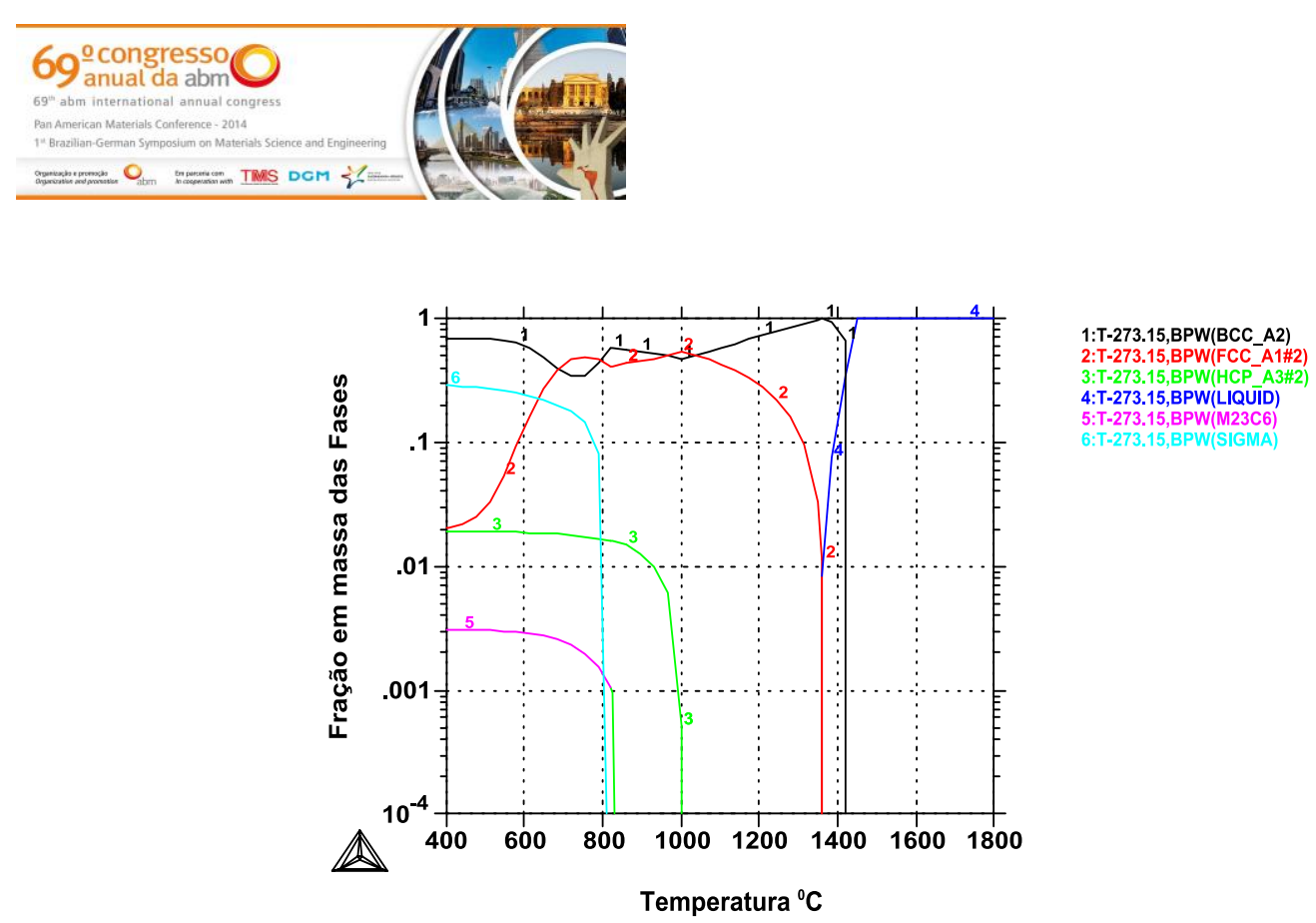

Figura 2 - Mapa de fases calculado com o programa Thermo-Calc $®$ aliado ao banco de dados TCFE. Nas ordenadas a escala é logarítmica (fração mássica das fases).

\subsection{Metodologia}

Com base nos mapas de fase calculados, para todos os ensaios as amostras foram tratadas termicamente sob vácuo em temperaturas de 800 e $900{ }^{\circ} \mathrm{C}$, por tempos de 1,8 e $24 \mathrm{~h}$, seguidos de têmpera em agua.

As amostras utilizadas para a metalografia e medidas de Ruido Magnetico de Barkhausen (MBN), tinham dimensões de $30 \times 30 \times 3 \mathrm{~mm}$, e foram retiradas com a face paralela à direção normal à direção de laminação. É interessante notar que a presença de textura cristalográfica pode influir no sinal obtido nas medidas de ruído magnético.

\subsubsection{Medições magnéticas de Barkhausen}

A medição de sinais MBN utilizaram uma excitação de onda senoidal magnética de $10 \mathrm{~Hz}$, e um campo magnético de $\pm 1,2 \times 10^{4} \mathrm{~A} / \mathrm{m}$. Uma bobina de captação, colocada perpendicularmente à amostra, detectando o sinal MBN a saída do sinal foi amplificado e a banda passante filtrada $(1-150 \mathrm{Khz})$. A frequência de amostragem aplicada foi de $400 \mathrm{Khz}$.

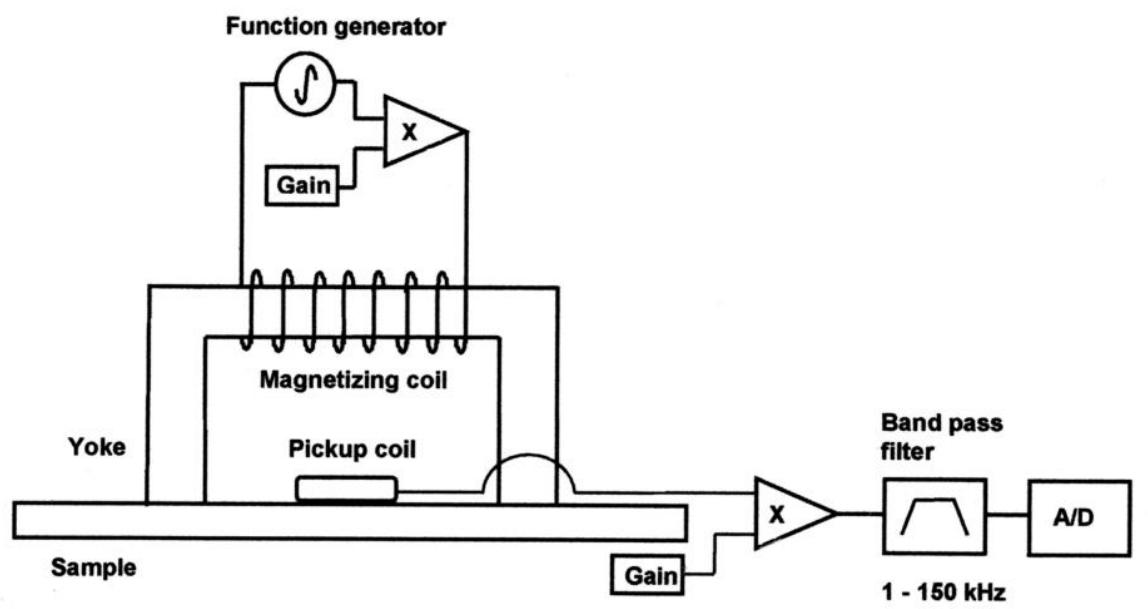

Figura 3 - Diagrama do esquema da medição de ruído magnético de Barkhausen (MBN).

\footnotetext{
* Contribuição técnica ao 69ำ Congresso Anual da ABM - Internacional e ao 14ํㅡㄹ ENEMET - Encontro Nacional de Estudantes de Engenharia Metalúrgica, de Materiais e de Minas, 21 a 25 de julho de 2014, São Paulo, SP, Brasil.
} 


\section{RESULTADOS E DISCUSSÃO}

\subsection{Caracterização das Amostras como Recebidas}

A microestrutura das amostras como recebidas é apresentada na figura 4. A estrutura é composta de ferrita e austenita, aparentemente na forma de lamelas alternadas. A Duplex como recebido apresenta ferrita e austenita. $O$ sinal característico de RMB da amostra como recebida é mostrado na figura 5, e será novamente referenciado posteriormente para comparação com os resultados obtidos após tratamento térmico.

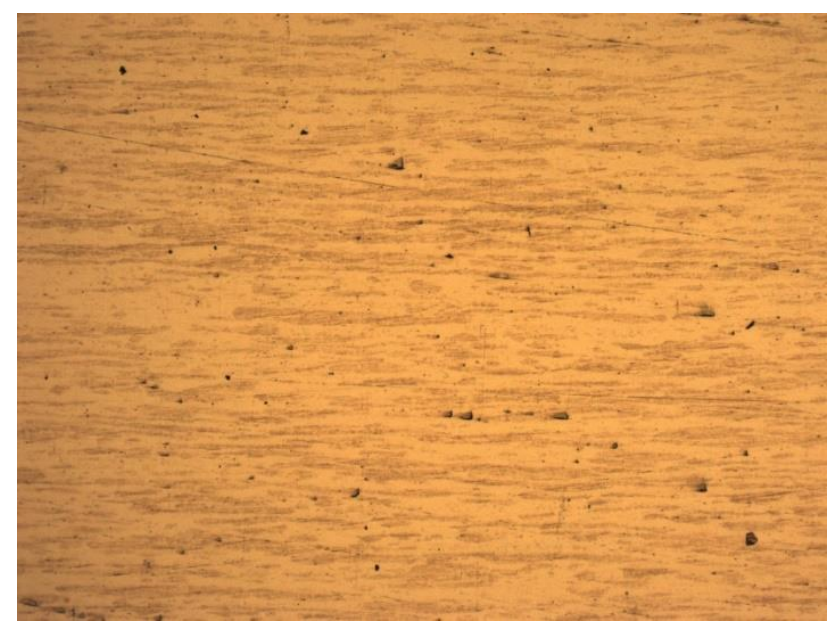

Figura 4 - Amostra como recebida.

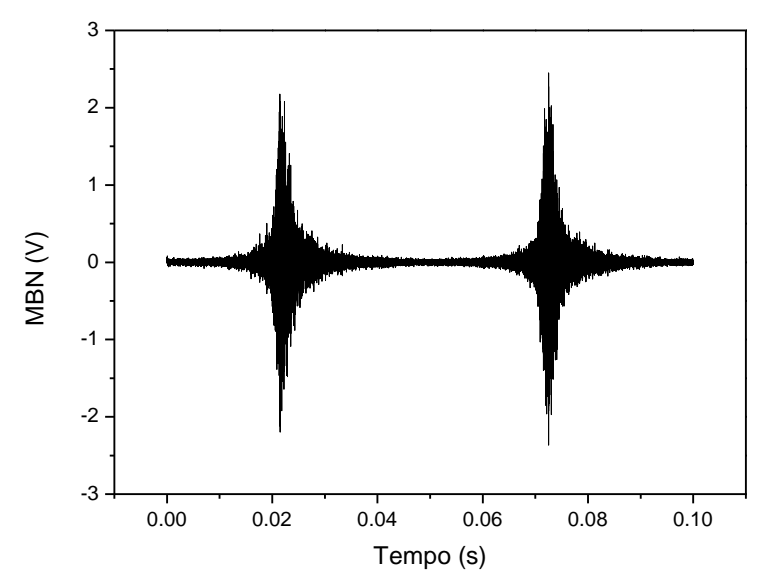

Figura 5 - sinal MBN como recebido

\subsection{Amostras após Tratamentos Térmicos}

As microestruturas das amostras submetidas a tratamentos térmicos isotérmicos são mostradas nas figuras 6 e 7. Pode-se notar em todas as amostras que a formação de fase sigma se dá por meio de consumo da fase ferrítica, seguindo a reação eutetóide $\alpha->\gamma+\sigma$. À medida que a reação avança a fração de fase ferromagnética (ferrita) diminui sensivelmente, e o que se espera dessa redução da fração de ferrita é que a intensidade do sinal de ruído Barkhausen também diminua sensivelmente, devido à menor fração de fase ferromagnética. Nota-se também que, nas amostras tratadas a $800^{\circ} \mathrm{C}$ em tempos mais longos, a reação encontra-se em estágio mais

\footnotetext{
* Contribuição técnica ao $69^{\circ}$ Congresso Anual da ABM - Internacional e ao 14ํㅡㄹ ENEMET - Encontro Nacional de Estudantes de Engenharia Metalúrgica, de Materiais e de Minas, 21 a 25 de julho de 2014, São Paulo, SP, Brasil.
} 

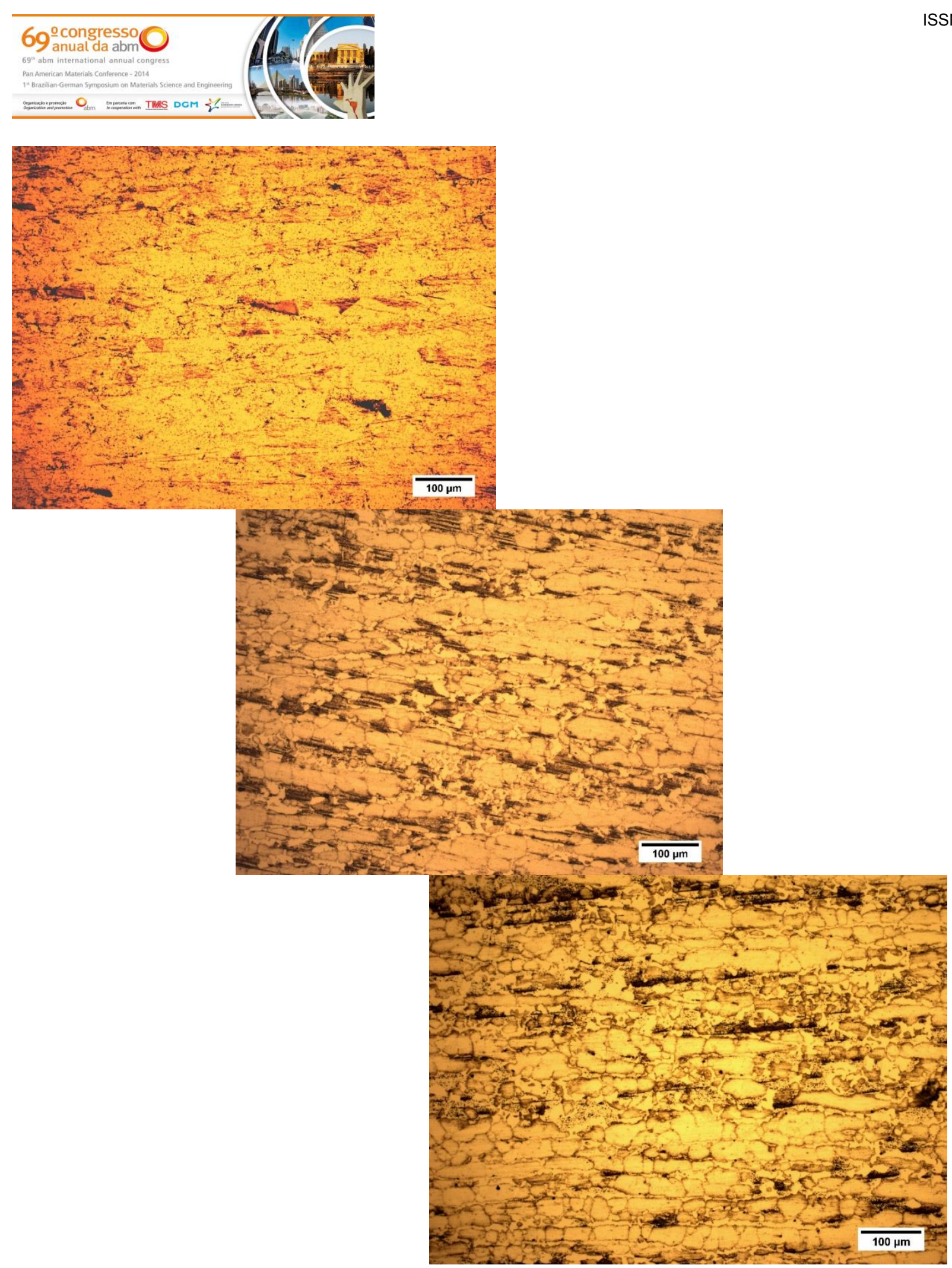

Figura 7 - Metalografias dos ensaios a $900^{\circ} \mathrm{C}$, a tempos de 1,8 e $24 \mathrm{~h}$.

\subsection{Ruído de Barkhausen}

Os sinais característicos de MBN das amostras como recebidas e após tratamento térmico são mostradas nas figuras 8 e 9 . Pode-se notar claramente que a intensidade do sinal de ruído magnético é muito maior na amostra como recebida do que nas amostras submetidas a tratamentos térmicos. A variação da intensidade RMS do sinal de ruído Barkhausen para as amostras tratadas termicamente é mostrada nas figuras 10 a 12. A queda de intensidade do sinal é muito mais acentuada nos estágios iniciais de tratamento, de forma que a queda proporcional

\footnotetext{
* Contribuição técnica ao 69 Congresso Anual da ABM - Internacional e ao 14ํㅡㄹ ENEMET - Encontro Nacional de Estudantes de Engenharia Metalúrgica, de Materiais e de Minas, 21 a 25 de julho de 2014, São Paulo, SP, Brasil.
} 


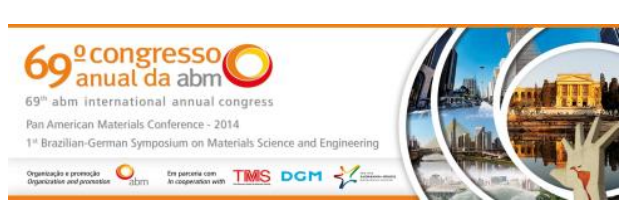

entre tempos mais longos de tratamento (por exemplo da amostra tratada por 8 horas para a amostra tratada por 24 horas) é muito mais tênue. Isso é um indicativo claro de que a reação de decomposição da ferrita em austenita e sigma está quase completa já com tempos de tratamento térmico da ordem de 8 horas.
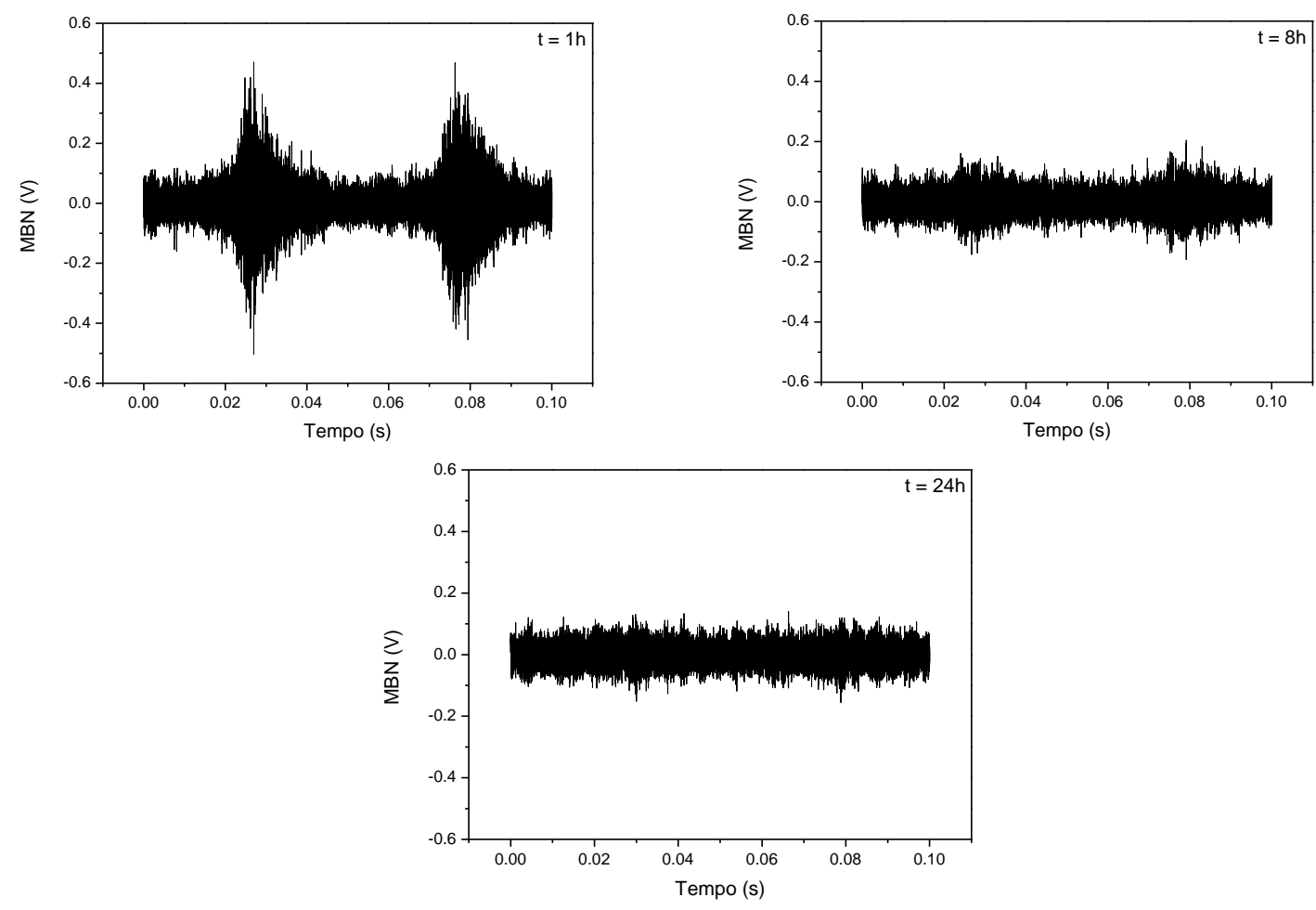

Figura 8 - Sinais de Barkhausen típicos a $800{ }^{\circ} \mathrm{C}$ no decorrer no tempo.
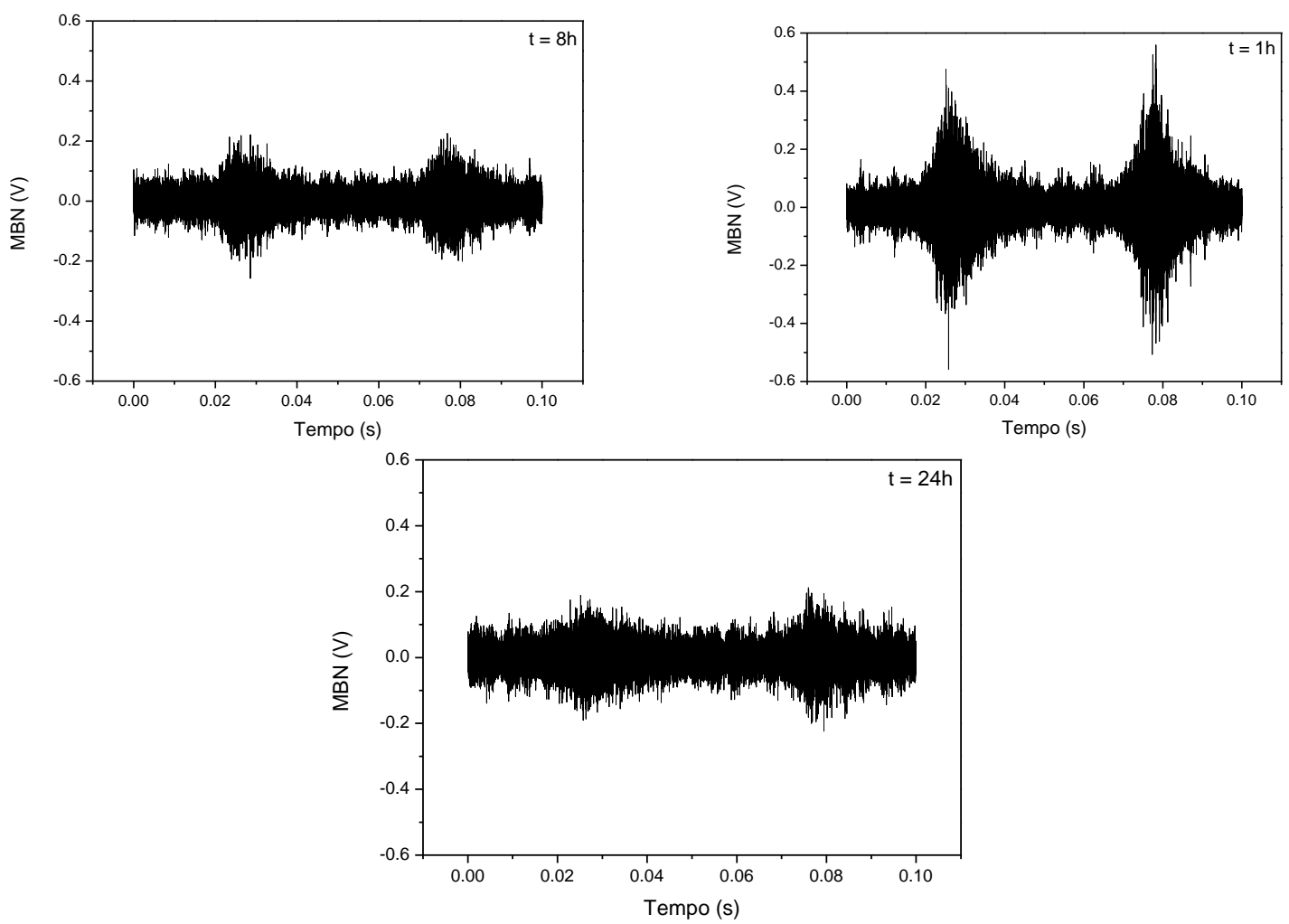

Figura 9 - Barkhausen a $900^{\circ} \mathrm{C}$ no decorrer no tempo.

* Contribuição técnica ao $69^{\circ}$ Congresso Anual da ABM - Internacional e ao 14ํㅡㄹ ENEMET - Encontro Nacional de Estudantes de Engenharia Metalúrgica, de Materiais e de Minas, 21 a 25 de julho de 2014, São Paulo, SP, Brasil. 
Uma comparação da variação de intensidade RMS do ruído Barkhausen nas amostras tratadas a $800^{\circ} \mathrm{C}$ e $900^{\circ} \mathrm{C}$ é mostrada na figura 10 a 12 .

Neste trabalho a voltagem RMS (root mean square) dá sinal de Barkhausen é definido como:

$R M S=\sqrt{\frac{\sum_{i} V_{I}^{2}}{n}}$

Onde o Vi é a voltagem de cada salto da sinal e n é número total dos eventos numa só medição.

Pode-se notar que A queda é menos acentuada na amostra tratada a $900^{\circ} \mathrm{C}$. Essa é provavelmente uma questão cinética. A formação da fase sigma pode ter sua cinética descrita em termos de uma curva em C. A amostra tratada a $900^{\circ} \mathrm{C}$, provavelmente encontrava-se pouco acima do nariz da curva, ao passo que a amostra tratada a $800^{\circ} \mathrm{C}$ encontrava-se pouco abaixo desta posição, obtendo-se assim uma cinética de formação mais acelerada a $800^{\circ} \mathrm{C}$.

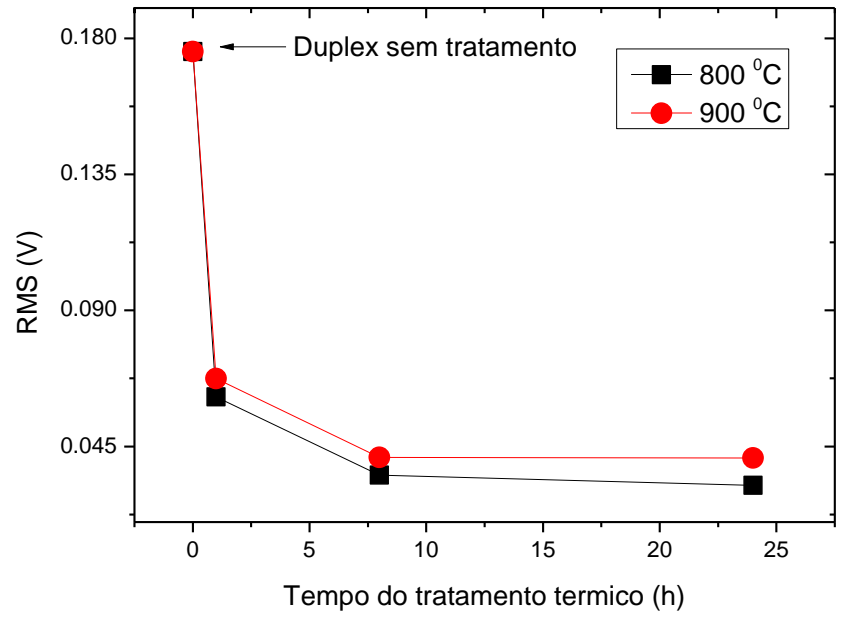

Figura 10 - Tratamentos térmicos de 800 e $900^{\circ} \mathrm{C}$ incluindo o material de início no sentido de laminação.

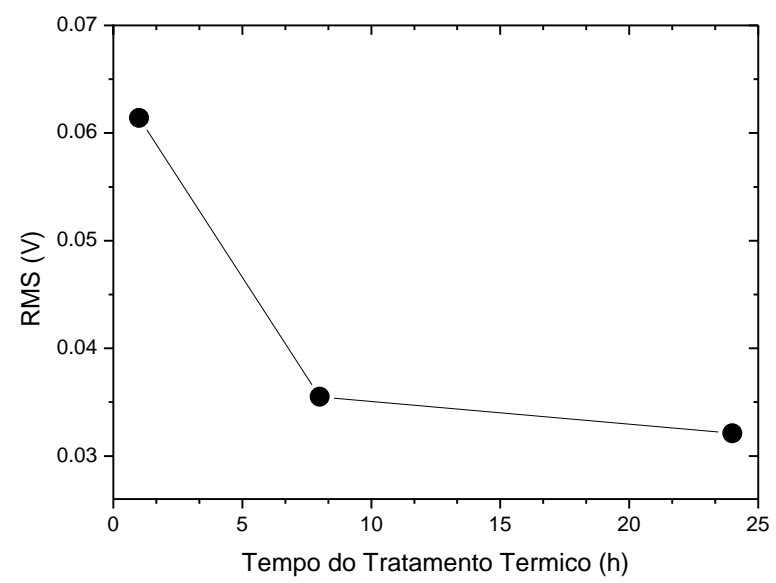

Figura 11 - Tratamentos térmicos a $800^{\circ} \mathrm{C}$, por tempos de 1 , 8 e 24 horas no sentido da laminação.

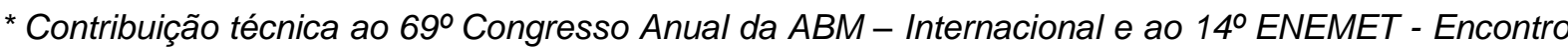
Nacional de Estudantes de Engenharia Metalúrgica, de Materiais e de Minas, 21 a 25 de julho de 2014, São Paulo, SP, Brasil.
} 

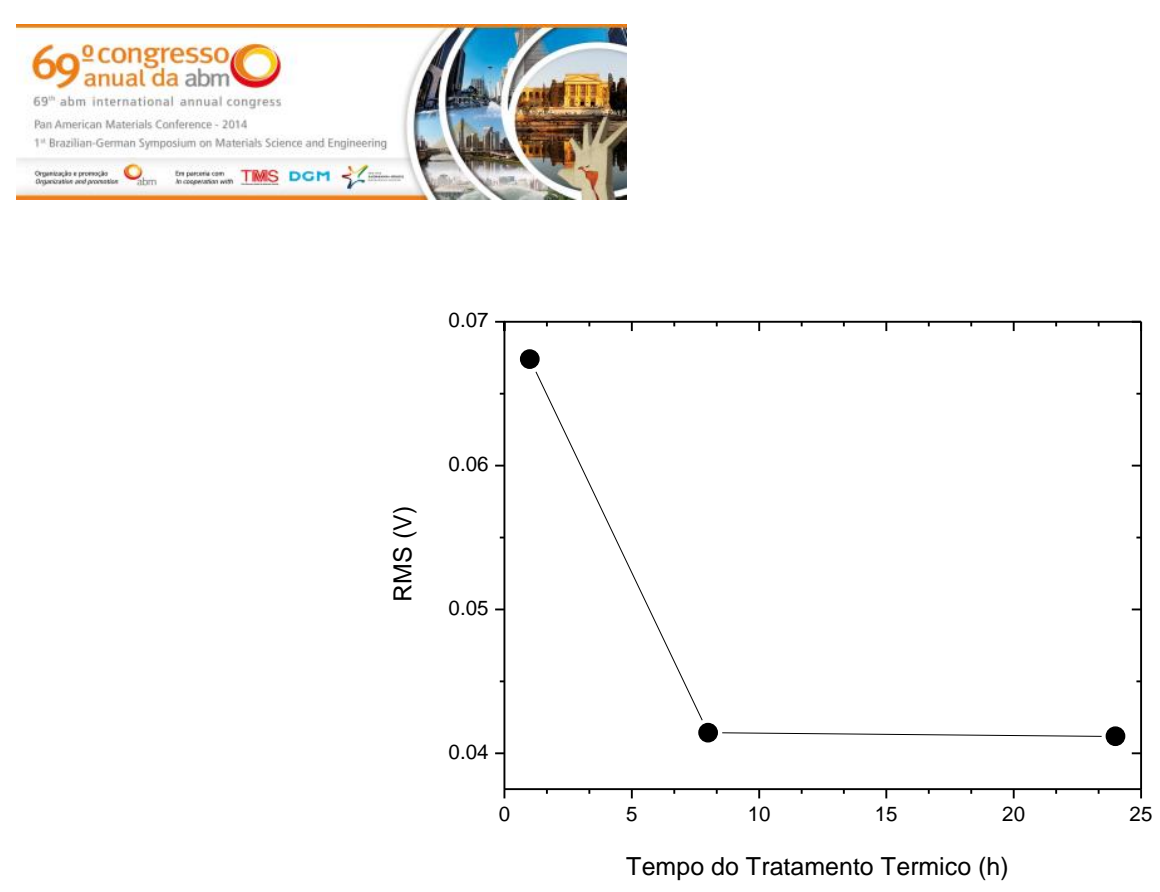

Figura 12 - Tratamentos térmicos a $900^{\circ} \mathrm{C}$, por tempos de 1, 8 e 24 horas no sentido da laminação.

Por meio da medida de fração volumétrica de ferrita nas amostras tratadas em vários tempos, é provável que se possa construir uma curva de calibração para fração de ferrita e fração de sigma em função da intensidade do sinal de ruído magnético. Para que essa curva de calibração possa ser adequadamente utilizada para se estimar a quantidade de fase sigma formada em um material sendo testado, é necessário que, na temperatura de trabalho, não haja formação de outras fases além de sigma e austenita, como por exemplo nitretos de cromo. Além da estimativa de quantidades de fases presentes, a correta calibração das curvas de ruído magnético com a microestrutura tornaria possível extrair das curvas de variação de intensidade RMS do ruído magnético uma curva que descreva a cinética da reação eutetóide, podendo-se assim fazer estimativas de vida útil de componentes submetidos a condições de trabalho nas quais pode ocorrer a formação de fases intermetálicas. Da mesma forma que se utiliza neste trabalho a fase sigma como objeto de estudo, poder-se-ia também aplicar o mesmo princípio para avaliação da formação de outras fases em outras condições de trabalho.

\section{CONCLUSÃO}

A evolução da fase sigma por tratamentos isotérmicos a $800^{\circ} \mathrm{C}$ e $900^{\circ} \mathrm{C}$ provocou 0 decréscimo da fase ferromagnética.

A fase sigma provocou o decréscimo do ruído magnético de Barkhausen MBN.

A formação de pequenos teores de fase sigma pode ser detectada de forma não destrutiva por MBN, o método é bastante sensível ao decorrer do tempo.

Pode-se montar uma curva de calibração da fase ferrita e sigma (paramagnética) para aplicações industriais em função da intensidade do MBN e da fração volumétricas das fases presentes.

\section{Agradecimentos}

Os autores agradecem às agências brasileiras de fomento à pesquisa (CNPq, CAPES-PROEX e FAPESP)

\footnotetext{
* Contribuição técnica ao 69ำ Congresso Anual da ABM - Internacional e ao 14ํㅡㄹ ENEMET - Encontro Nacional de Estudantes de Engenharia Metalúrgica, de Materiais e de Minas, 21 a 25 de julho de 2014, São Paulo, SP, Brasil.
} 


\section{REFERÊNCIAS}

1 Blaow M, Evans JT, Shaw BA. Magnetic Barkhausen noise: the influence of microstructure and deformation in bending. Acta Materialia, 2005; 53: 279-287.

2 Alberteris CM, Capó SJ, Pérez BJ, Padovese LR. Characterization of the elastic-plastic region in AISI/SAE 1070 steel by the magnetic barkhausen noise. NDT\&E International, 2008; 41: 656-659.

3 Jiles DC. Dynamics of domain magnetization and the Barkhausen effect. Czechoslovak Journal Physics, 2000; 50: 893-988. 